\title{
Observations of Rising Tone Chorus Emissions at Indian Low Latitude Ground Station and Their Generation Mechanism
}

\author{
S. B. Singh, K. K. Singh and A. K. Singh \\ Atmospheric Research Lab., Department of Physics, \\ Banaras Hindu University, Varanasi-221005, India.
}

\begin{abstract}
Chorus emissions are the most common form of very low frequency (VLF) emissions in the Earth's magnetosphere which typically consist of a series of rising tones generated near the magnetic equator, excited by energetic electrons injected into the inner magnetosphere. In the present study, observation of chorus emissions recorded at Indian low latitude ground station Jammu (geomag. lat., $19^{0} 26^{\prime} \mathrm{N} ; L=1.17$ ) during a geomagnetic quiet period on 24 February, 1999 is reported. The spectral analysis of recorded chorus emissions shows that each chorus element originates from the upper edge of the underlying hiss band. The observed mean chorus element parameters are as follows: lower band frequency $f_{\min }=1.2 \mathrm{kHz}$, upper band frequency $f_{U B}=1.96 \mathrm{kHz}$, frequency sweep rate $d f / d t=1.14 \mathrm{kHz} / \mathrm{s}$ and repetition period $T=2.5 \mathrm{~s}$. To explain the observed dynamic spectra of these chorus emissions, a possible generation mechanism is presented based on the recent nonlinear theory. It is observed that the seeds of chorus emissions grow from the saturation level of the whistler-mode instability at the equator and then propagate away from the equator as a result of a nonlinear growth mechanism that depends on the wave amplitude. On the basis of this theory, frequency sweep rate of chorus emission is computed and compared with that of our experimentally observed values, which shows, in general, a good agreement.
\end{abstract}

Keywords: Chorus emissions, Whistler-mode waves, Wave particle interaction.

\section{Introduction}

Chorus is known as coherent electromagnetic waves and consists of a series of rising tones near the magnetic equator, excited by energetic electrons from several $\mathrm{keV}$ to tens of $\mathrm{keV}$ injected into the inner magnetosphere (Helliwell, 1965; Tsurutani and Smith, 1974; Kasahara et al., 2005). Being a plasma wave mode of particular geophysical interest, chorus is believed to play a major role in the evolution of energetic electron distributions within the magnetosphere. Chorus typically comprises repeated narrowband monochromatic signals of short duration $(\sim 0.1 \mathrm{~s})$ with rising frequency and occurs at frequencies below the local electron gyrofrequency (Omura et al., 2009). The prevailing theory of VLF chorus says that chorus is produced by cyclotron resonance interaction between electrons and whistler-mode waves. Theoretical work on the cyclotron resonance interaction has been advanced to include a self-consistent solution of the problem (Nunn, 1974; Molving et al., 1988). However, the most useful theoretical treatment is the phenomenological model of Helliwell (1967). Although this model is not self-consistent, it is unique (Sazhin and Hawakawa, 1992) that it provides a procedure by which wave data can be 
compared to the cyclotron-resonance theory to determine if a given wave emission could have been produced by this mechanism (Skoug et al., 1996). In terms of cyclotron resonance interaction, cold plasma cyclotron interaction can produce the lower-frequency portions of the observed chorus risers from $1 \mathrm{kHz}$ to $2.5 \mathrm{kHz}$, while warm plasma model is required to produce $>2.5 \mathrm{kHz}$. The warm plasma model assumes a two-component plasma, with an isotropic cold component and a Maxwellian warm component. The effect of the warm component is to change the wave dispersion relation, allowing the production of the higher frequency risers (Skoug et al., 1996).

Trakhtengerts $(1995,1999)$ proposed a generation mechanism of chorus based on a model of a backward wave oscillator and nonlinear wave growth. Nonlinear models are commonly used to describe the properties of discrete emissions which are believed to be generated through coherent-gyroresonance interaction with electrons (Helliwell, 1967). Nonlinear theories approach generally requires the use of numerical simulation to provide solutions to the nonlinear equation of motion (Inan, 1977). The advantage of this approach has been the ability to include the effects of coherent phase bunching of the electrons, which has been believed to play a primary role in the generation of coherent wave forms such as chorus and discrete emissions (Helliwell and Inan, 1982). In all of these previous studies, a coherent wave is assumed as a seed of chorus elements, while the whistler-mode instability driven by temperature anisotropy gives a spectrum of waves that have positive growth rates (Omura and Summers, 2004). The essential problem was how the coherent chorus elements with variable frequency are excited in the equatorial region of the magnetosphere where the inhomogeneity of the magnetic field is very small. Recently, a mechanism of the rising chorus emission has been accepted in terms of nonlinear theory due to the formation of electromagnetic electron hole in the velocity phase space (Omura et al., 2008, 2009). In this mechanism, Omura et al. (2008) derived the relativistic second-order resonance condition for a chorus element with variable frequency. The relation between the wave amplitude and frequency sweep rate in the generation region of chorus emissions is demonstrated in a full particle electromagnetic simulation and these simulations show that seeds of chorus emissions with rising tones are formed in a localized region near the magnetic equator (Omura et al., 2009). Singh et al. (2010) applied successfully the nonlinear theory of Omura et al. (2008) to explain the observation of whistler-mode chorus emissions recorded at the Indian Antarctic station, Maitri (lat $=70^{\circ} 46^{\prime} \mathrm{S}$, long $=11^{0} 50^{\prime} \mathrm{E}, L=4.5$ ), during a quiet period on 5 February, 2001. Hikishima and Omura (2012) performed a self-consistent electromagnetic particle simulation to analyze whistler-mode triggered emissions in the magnetosphere and confirmed the optimum amplitude of triggering waves for rising-tone emissions. Recently, Tao et al. (2012) studied the theoretical predictions for chorus frequency sweep rates by Helliwell (1967) and Trakhtengerts (1995) and compared with observations from the THEMIS satellites and a previously published dataset from the Cluster satellites. They also extended the theories to use a general magnetic field model to include the effects of magnetic local time and geomagnetic activity, and showed that both theories give the same dependence of the frequency sweep rate on background plasma parameters. Still it is a subject of study as to what determines the frequency sweep rate of a chorus element. Thus, the detailed generation process of chorus emissions remains to be fully identified, particularly those observed at low latitudes. To complete this study the present study has been initiated to explain the chorus emissions observed at low latitude. The observation of chorus emissions at low latitude ground station Jammu are unusual in the sense that most of reported chorus emissions observed from either satellites (Cornilleau-Wehrlin et al., 1978; Hattori et al., 1991; Santolik and Gurnett, 2003) 
or from high latitude stations. An understanding of the generation mechanism of these chorus emissions observed during daytime at our low latitude ground station Jammu would be most useful for inferring the properties of high energy trapped electrons.

In this paper, we have explained the generation mechanism and spectral analysis of observed some typical rising tone chorus emissions at an Indian low latitude ground station Jammu (geomag. lat., $19^{0} 26^{\prime} \mathrm{N} ; L=1.17$ ) during a geomagnetic quiet period on $24^{\text {th }}$ February, 1999. The experimental setups as well as observations of chorus emissions with details of their dynamic spectra are presented in Section 2. The generation mechanism of the observed chorus emission based on the recent nonlinear theory (Omura et al., 2009) has been explained in Section 3. Section 4 presents the results and discussions of observed chorus emissions and their computed frequency sweep rate based on the presented generation mechanism. Finally the conclusion of the study is presented in Section 5.

\section{Experimental Observations}

The VLF data reported in this study were recorded at a low latitude Indian ground station Jammu (geomag. lat., $19^{0} 26^{\prime} \mathrm{N} ; L=1.17$ ). The signals were detected on the standard VLF observation equipment consisting of a T-type antenna which is 25 - meter long in vertical and 6 meter horizontally and $3.2 \mathrm{~mm}$ in diameter, having a bandwidth of $50 \mathrm{~Hz}-15 \mathrm{kHz}$. The VLF data recorded on magnetic tapes were analyzed using a software 'Raven' developed under Cornell Lab. of Ornithology Bioacoustics research program, USA and installed at Atmospheric Research Lab, Department of Physics, Banaras Hindu University, Varanasi. The software program can be used for the acquisition, visualization, measurement and analysis of acoustic as well as electromagnetic waves.

We have analyzed the VLF data recorded at Jammu during six months from January, 1999 to June, 1999 using the software Raven to study the various types of VLF emissions. During the routine recording of VLF waves at Jammu, we observed some interesting events of rising tone chorus emissions on $24^{\text {th }}$ February 1999 which have been presented in this study. The observed peculiar chorus emissions events started at 12:10:30 IST (Indian Standard Time) and the whole observation period of chorus events continued for about 30 minutes. Four typical sets of examples of chorus emissions observed at Jammu on $24^{\text {th }}$ February 1999 are shown in Figure 1. From the frequency time spectrogram of Figure 1(A) and 1(B), the hiss band is seen as an unstructured signal below about $1.2 \mathrm{kHz}$, and the discrete rising tones are chorus emissions. It is clearly seen that each chorus element originates from the upper edge of the underlying hiss band. Figure 1A contains four rising chorus emissions with mean upper cut-off frequency of $1.55 \mathrm{kHz}$ and mean lower cut-off frequency of $1.08 \mathrm{kHz}$. The mean frequency sweep rate $(d f / d t)$ of these chorus elements is $1.15 \mathrm{kHz} / \mathrm{s}$. The repetition period $(T)$ of these chorus elements is $2.3 \mathrm{~s}$. Figure $1 \mathrm{~B}$ contains three chorus emissions with mean upper cut-off frequency of $1.75 \mathrm{kHz}$ and mean lower cut-off frequency of $1.28 \mathrm{kHz}$. The mean $d f / d t$ of these chorus elements is $1.01 \mathrm{kHz} / \mathrm{s}$. Figure 1C contains four chorus emissions with upper cut-off frequency of $1.96 \mathrm{kHz}$ and lower cut-off frequency of $1.1 \mathrm{kHz}$ having the mean $d f / d t=1.1 \mathrm{kHz} / \mathrm{s}$. Figure $1 \mathrm{D}$ contains three chorus emissions with upper cut-off frequency of $1.78 \mathrm{kHz}$ and lower cut-off frequency of $1.2 \mathrm{kHz}$ having the mean $d f / d t=1.2 \mathrm{kHz} / \mathrm{s}$. Different frequencies can be generated in different subregions along the field line in the case of such an extended source. The horizontal lines in Figure 1 are artificial noises generated by high power transmission line signals. Figure 1 shows that the upper cut-off frequencies for different chorus events are different, so we have calculated the mean chorus element parameters which are as follows: lower band frequency $f_{\min }=1.1 \mathrm{kHz}$, 

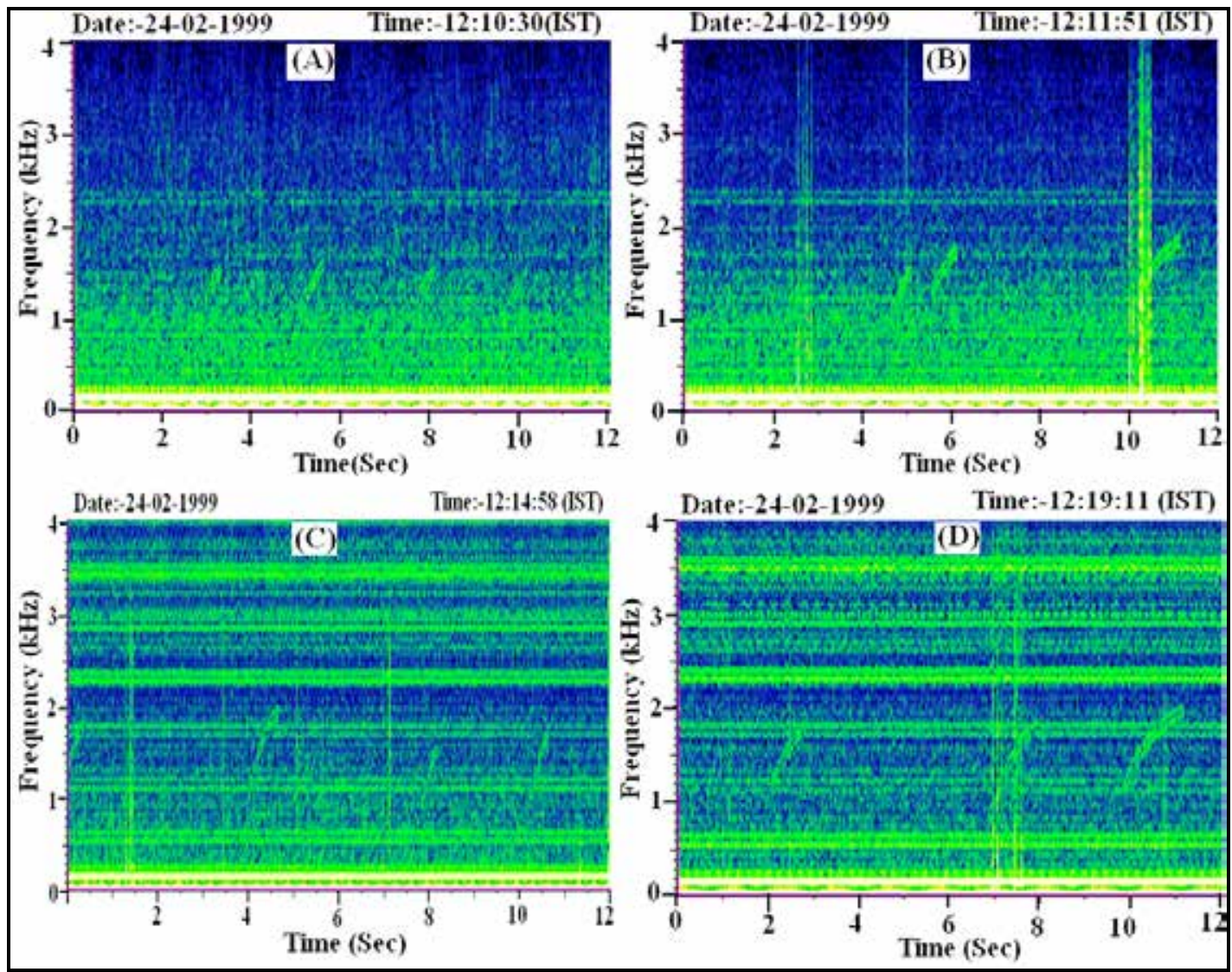

Figure 1: Typical examples of chorus emissions observed at Jammu on $24^{\text {th }}$ February, 1999.

upper band frequency $f_{U B}=1.96 \mathrm{kHz}$, frequency sweep rate $d f / d t=1.14 \mathrm{kHz} / \mathrm{s}$ and repetition period $T=2.5 \mathrm{~s}$. Most of chorus emissions are found to exhibit a moderate $d f / d t$ of the order of $1 \mathrm{kHz} / \mathrm{s}$, but there were no chorus emissions with falling or constant frequency tones and for the rising tones $d f / d t$ is in a range from 0.8 to $1.5 \mathrm{kHz} / \mathrm{s}$ (Hayakawa, 1993). These typical chorus emissions were observed on a quiet geomagnetic day of $24^{\text {th }}$ February 1999 with geomagnetic activity $\sum \mathrm{Kp}=17$. and Dst index $=-28 \mathrm{nT}$. The variation of Dst index, $\mathrm{AE}$ index and $\mathrm{K}_{\mathrm{P}}$ index with time during 23-25 February 1999 are shown in Figs. 2A, B and C, consecutively in which the time of received VLF chorus emissions is also marked.

\section{Generation Mechanism}

A number of studies have been done to know the process governing the mechanism of chorus emissions (Helliwell, 1967; Omura and Matsumoto, 1982; Trakhtengerts, 1999; Singh and Ronnmark, 2004; Katoh and Omura, 2007; Omura et al., 2008; Singh et al., 2010). It is generally accepted that chorus waves can be excited by cyclotron resonance with anisotropic 10- 
$100 \mathrm{keV}$ electrons. Nunn (1974) and Nunn et al. (1997) simulated the generation process of chorus emissions by a Vlasov-hybrid simulation using a coherent wave as seed of a chorus element. Omura and Matsumoto (1982) performed simulation of whistler-mode wave electron interaction demonstrating the basic process of wave amplification via nonlinear resonant currents. Trakhtengerts $(1995,1999)$ proposed a generation mechanism of chorus based on a model of a backward wave oscillator and nonlinear wave growth.

In all of these previous studies, a coherent wave is assumed as a seed of chorus elements while the whistler-mode instability driven by temperature anisotropy gives a spectrum of waves that have positive growth rate (Omura and Summer, 2004). By direction finding measurements Burton and Holzer (1974) found that wave normal angles for the dayside chorus are highly concentrated to a range less than $25^{\circ}$. Hayakawa (1993) has also found by direction finding measurements that the wave normals of the rising tone chorus take very small angles $\left(5^{0}-20^{0}\right)$. We have considered the wave normal angle $\theta=0^{0}$ for the present study of chorus emission. For the whistler-mode wave propagating parallel to the static magnetic field, the cold plasma dispersion relation is written as (Stix, 1992)

$$
c^{2} k^{2}=\omega^{2}+\frac{\omega \omega_{p e}^{2}}{\Omega_{e}-\omega}
$$

where $c=$ speed of light, $k=$ wave number, $\omega_{p e}=$ electron plasma frequency, $\Omega e=$ electron cyclotron frequency. The wave phase velocity $V_{p}$ is given as

$$
V_{p}=\frac{\omega}{k}=c \delta \xi
$$

where $\delta$ and $\xi$ are dimensionless parameters defined as (Omura et al., 2007)

$$
\delta^{2}=1-\frac{\omega^{2}}{c^{2} k^{2}}
$$

and

$$
\xi^{2}=\omega \frac{\left(\Omega_{e}-\omega\right)}{\omega_{p_{e}}^{2}}
$$

Differentiating (1) with respect to $\omega$, we get

$$
2 c^{2} k^{2} \frac{\partial k}{\partial \omega}=2 \omega+\frac{\Omega_{e} \omega_{p e}^{2}}{\left(\Omega_{e}-\omega\right)^{2}}
$$

We assume a coherent whistler-mode wave with amplitude $B_{\omega}$ and phase angle $\Psi . B_{\omega}$ is a slowly varying function of position $h$ and time $t$ and that the phase $\Psi$ varies smoothly in space and time. The frequency $\omega$ and the wave number $k$ are defined as $\omega=\partial \psi / \partial t$ and $k=-\partial \psi / \partial h$. Since we change the order of differentiation in the second-order derivative of $\Psi$ by $h$ and $t$ i.e. 


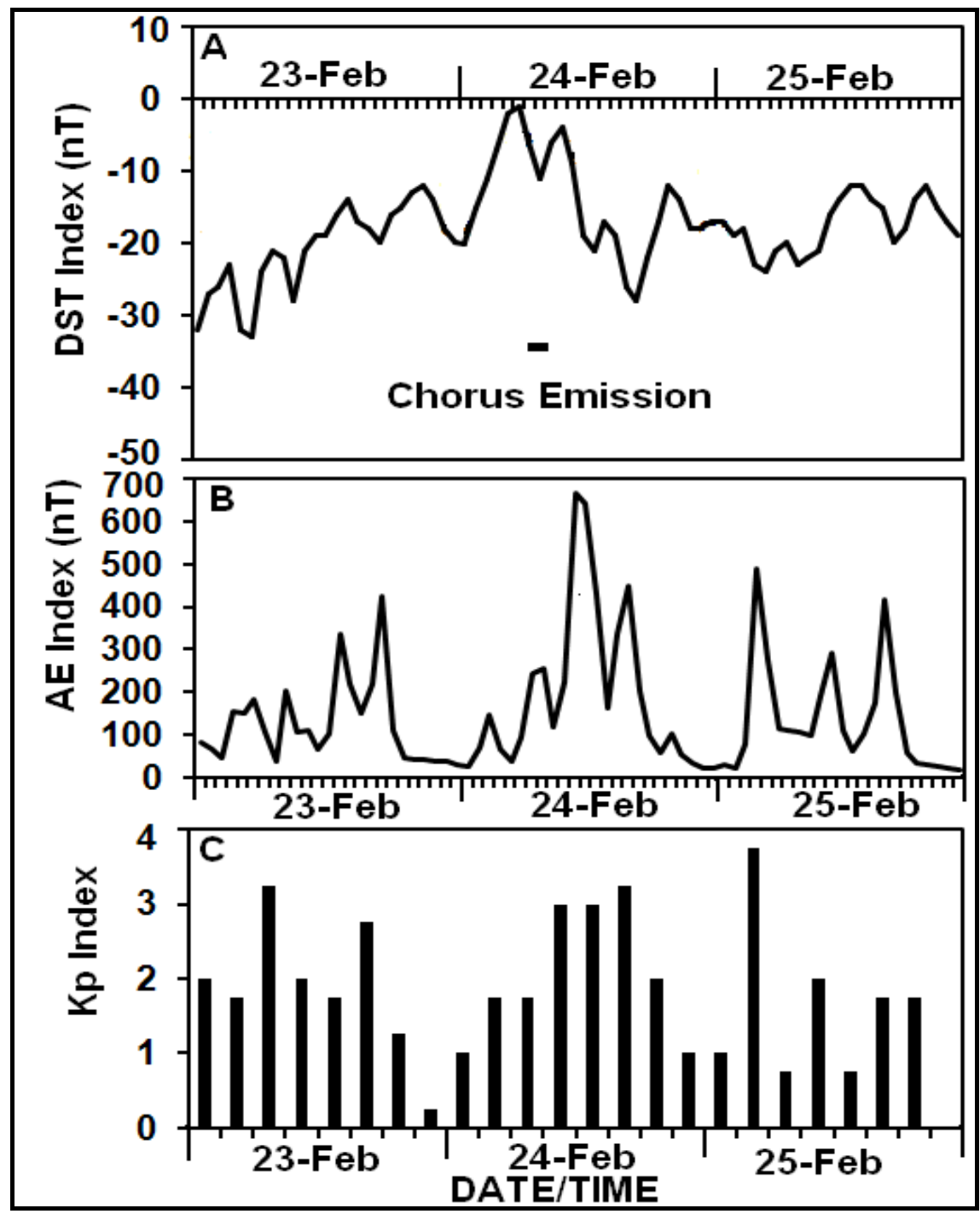

Figure 2: Variation of (A) Dst index, (B) AE index and (C) $K_{P}$ index during 23-25 February 1999. The observation time of received VLF chorus emissions is also marked.

$\partial^{2} \psi / \partial h \partial t$, we have

$$
\frac{\partial k}{\partial t}=-\frac{\partial \omega k}{\partial h}
$$


We again suppose that an electron moves with parallel $V_{I I}$ and perpendicular $V_{\perp}$ velocities, so we define the resonance velocity $V_{R}$ that satisfies Doppler cyclotron resonance condition,

$$
V_{R}=\frac{1}{k}\left(\omega-\frac{\Omega_{e}}{\gamma}\right)
$$

where $\gamma=\left[1-\left(V_{\mathrm{II}}^{2}+V_{\perp}^{2}\right) / c^{2}\right]^{-1 / 2}$, if $V_{I I}=V_{R}$, the electron resonates with the wave and 'sees' a constant phase of the wave field.

From Maxwell's equations retaining the displacement current which has been neglected in previous studies of whistler-mode waves we obtain the wave equation for the amplitude $B_{\omega}$ of the wave magnetic field in the following form (Omura et al., 2008, 2009)

$$
\frac{\partial B_{\omega}}{\partial t}+V_{g} \frac{\partial B_{\omega}}{\partial h}=-\frac{\mu_{o} V_{g}}{2} J_{E}
$$

where $\mu_{o}$ and $J_{E}$ are the vacuum permeability and the component resonant current parallel to the wave electric field. The resonant current is formed by the interaction of resonant electrons with the wave. The frequency $\omega$ is constant in the frame of reference moving with the group velocity $V_{g}$ as expressed by the equation

$$
\frac{\partial \omega}{\partial t}+V_{g} \frac{\partial \omega}{\partial h}=0
$$

Equation (8) describes the growth of the wave amplitude $B_{\omega}$ and we can write alternatively

$$
\frac{\partial B_{\omega}}{\partial t}=\omega_{i} B_{\omega}
$$

where $\omega_{i}$ is wave growth rate. The nonlinear growth of a chorus element near the equator is controlled by the frequency rate or the time derivative of the frequency $\partial \omega / \partial t$. In equation (8) a negative value of $-J_{E}$ contributes to wave growth, while $J_{B}$ only modifies the dispersion relation. The $J_{B}$ is resonant current which is anti-parallel to the wave magnetic field and causes the nonlinear growth rate. According to the linear theory, at the equator there is a finite spectrum of whistler-mode waves being generated by the temperature anisotropy of the resonant electrons. From these waves with different rates of phase variation, a coherent wave can develop with phase variation $\partial \omega / \partial t$ that maximizes the value of $-J_{E}$ at the equator for inhomogeneity ratio $S$ $=-0.41$. Higher wave amplitudes are thereby achieved. The parameter $S$ is the inhomogeneity ratio given by (Omura et al., 2008)

$$
S=-\frac{1}{\omega_{t}^{2} \delta^{2}}\left\{\gamma\left(1+\frac{-V_{R}}{V_{g}}\right)^{2} \frac{\partial \omega}{\partial t}+\left[\frac{k \nu_{\perp}^{2}}{2 \Omega_{e}}-\left(1+\frac{\delta^{2}}{2} \frac{\Omega_{e}-\gamma \omega}{\Omega_{e}-\omega}\right) V_{R}\right] \frac{\partial \Omega_{e}}{\partial h}\right\}
$$


$S$ is a ratio of inhomogeneity term to the oscillatory term in the pendulum equation ( $\mathrm{S}=0)$. When $|\mathrm{S}|>1$, there is no equilibrium state, and when $|\mathrm{S}|<1$, there is a small amplitude solution that oscillates with the modified trapping frequency. When a chorus element with a rising frequency propagates northward away from the magnetic equator and interacts with resonant electrons moving in the opposite direction, $S$ takes a negative value (Omura et al., 2008). By setting $\frac{\partial \Omega_{e}}{\partial h}$ $=0$ in equation (11), the inhomogeneity ratio $S$ at the equator is given by (Omura et al., 2008)

$$
S_{E Q}=-\frac{\gamma}{\omega_{t}^{2} \delta^{2}}\left(1+\frac{-V_{R}}{V_{g}}\right)^{2} \frac{\partial \omega}{\partial t}
$$

Setting $S_{E Q}=-0.4$ in equation (12), we get a condition for the maximum nonlinear growth of the wave as

$$
\frac{\partial \omega}{\partial t}=0.4 \frac{\omega_{t}^{2} \delta^{2}}{\gamma}\left(1+\frac{-V_{R}}{V_{g}}\right)^{-2}
$$

Using $\omega_{t}^{2}=k V_{\perp} \Omega_{\omega}$ where $\Omega_{\omega}=e B_{\omega} / m_{0}$, we can again rewrite (13) as (Omura et al., 2008),

$$
\frac{\partial \omega}{\partial t}=\frac{0.4 \delta}{\gamma \xi} \frac{V_{\perp}}{c} \frac{\omega}{\Omega_{e}}\left[1+\frac{-V_{R}}{V_{g}}\right]^{-2} \frac{B_{W}}{B_{O}} \Omega_{e}^{2}
$$

where from Eqs. (2) and (7),

$$
V_{R}=c \delta \xi\left(1-\frac{\Omega_{e}}{\gamma \omega}\right)
$$

and

$$
1+\frac{-V_{R}}{V_{g}}=1+\delta^{2}\left(\xi^{2}+\frac{\Omega_{e}}{2\left(\Omega_{e}-\omega\right)}\right)\left(\frac{\Omega_{e}}{\gamma \omega}-1\right)
$$

Thus, the nonlinear wave growth can continue over some distance from the equator to generate chorus elements. Around the equator where the flux of the resonant energetic electrons maximizes, the resonant current forms because of the electron hole in the velocity phase space. Among the whistler-mode waves with different phases, it is the modes that satisfy the secondorder resonance condition with $\mathrm{S} \sim-0.4$ that undergoes nonlinear wave growth to form chorus emissions (Omura et al., 2008). 
To test this theory of the nonlinear generation mechanism, we have computed the frequency sweep rate of chorus elements using equations (13) - (16), and compared this with our experimentally observed values.

\section{Results and Discusions}

Some interesting events of rising tone chorus emissions observed on a quiet day $24^{\text {th }}$ February 1999 at a low latitude ground station Jammu have been reported. From about 30 minutes of chorus observations, we have selected four different intervals of chorus data for detailed study. The detailed spectral analysis of chorus events suggests that these emissions are likely to have originated from the underlying hiss band (Hattori et al., 1991). Hattori and Hayakawa (1994) also suggested that a chorus event is triggered from a wavelet existing at the upper edge of the hiss band through a coherent wave-particle interaction. The most frequent observations of a chorus events by satellites near the geomagnetic equtor (Tsurutani and Smith, 1974; Bruits and Helliwell, 1976) support the idea that the source of chorus emissions is most likely near the equtorial region. Lauben et al. (2002) studied various source characteteristics of chorus emisions and indicated the source region near the magnetic equator.

The upper boundary frequency (UBF) method (Smirnova, 1984) has generally been used to find the location of the ground observed VLF emisions (Singh et al., 2004; Singh et al., 2009). The upper boundary frequency of the ground obseved VLF emissions is determined on the assumption of a dipolar geomagnetic field configuration by the half electron gyrofrequency region, irrespctive of the observation station. The L-value of the source is then computed with the help of the ralation (Smirnova, 1984)

$$
L=\left(440 / f_{U B}\right)^{1 / 3}
$$

where $f_{U B}$ is the upper boundary frequency of the emissions in $\mathrm{kHz}$. Using equation (17) and observed parameters, the value of the source region of the chorus emission observed at Jammu, is found to be $L_{\text {source }}=5.9$. A source moving across L shells in the equatorial plane could also naturally explain the frequency shifts due to the variation of the local electron cyclotron frequency in the generation region. This supports the possibility for the VLF emissions to propagate down to the ionosphere is in ducted propagation $(\theta=0)$ at high latitudes (higher Lvalues). In this case of high-latitude penetration through the ionosphere it is possible for the waves to propagate in the waveguide towards lower latitudes and are received at low latitude station Jammu (Singh et al., 2004; Singh et al., 2009).

We have examined the generation mechanism with the help of nonlinear theory by using equations (13)-(16). The sources of chorus elements most probably have a finite length along the field line. The L-value of the source region is $L_{\text {source }}=5.9$ and the equatorial electron gyrofrequency is $f_{B}=4.2 \mathrm{kHz}$ for the rising tone chorus emissions observed at Jammu. According to the empirical equatorial electron density profile model of Carpenter and Anderson (1992) the electron density at $L_{\text {source }}=5.9$ is taken as $\sim 9$ electron $\mathrm{cm}^{-3}$ and the corresponding plasma frequency $\sim 27 \mathrm{kHz}$. Considering a frequency $f=f_{B} / 3$, we find from the dispersion relation that the wave number $k \sim 0.39 \mathrm{~km}^{-1}$. The wave group velocity $V_{g}=2.33 \times 10^{7} \mathrm{~ms}^{-1}$, the electron parallel velocity $V_{I I}=6.99 \times 10^{7} \mathrm{~ms}^{-1}$ and the electron perpendicular velocity $V_{\perp}=2.2 \times$ $10^{7} \mathrm{~ms}^{-1}$. We find the dimensionless parameters $\delta^{2}$ and $\xi^{2}$ from equations (3) and (4) as 0.99 and 0.0054 respectively. The value of $\gamma=1.03$ and $\Omega_{\omega}=e B_{\omega} / m_{0}=26.37$, considering that 
the maximum value $B_{\omega}=150 p T$ observed by Cluster spacecraft (Santolik et al., 2004). Using equation (16) we computed $\left[1+\left(-V_{R} / V_{g}\right)\right]=2.41$. Substituting all the parameters in equation (14) we find $\partial \omega / \partial t=15.27 \mathrm{kHz} / \mathrm{s}$ hence frequency sweep rate $\partial f / \partial t=2.43 \mathrm{kHz} / \mathrm{s}$ which is very much comparable to our experimentally observed result of $1.14 \mathrm{kHz} / \mathrm{s}$. Thus our results confirm that the nonlinear theory of generation mechanism of chorus emissions is consistent with the analysis of chorus emission observed at Indian low latitude station Jammu. Omura et al. (2008) successfully applied this theory to reproduce the formation of rising tone chorus elements propagating away from the equator using a self-consistent particle simulation (Katoh and Omura, 2006). Hikishima et al. (2009) successfully performed an electromagnetic full-particle simulation to study the generation mechanism of VLF whistler-mode chorus emissions in the equatorial region of the magnetosphere using a parabolic variation of the static magnetic field. Singh et al. (2010) successfully applied the nonlinear theory to explain the observation of whistler-mode chorus emissions recorded at the Indian Antarctic station, Maitri during a quiet period on 5 February, 2001. Further experimental and theoretical studies of VLF chorus emissions using some more long data sets recorded at low latitudes are required for a complete understanding of the generation mechanism of chorus emissions observed at low latitudes.

\section{Conclusions}

The spectral analysis of chorus events observed at an Indian low latitude ground station Jammu during a geomagnetic quiet period suggests that these emissions are likely to have originated from the upper edge of underlying hiss band. The source region of the chorus emissions obderved at Jammu is found to be $L_{\text {source }}=5.9$. This suggests the possibility that these emissions may have propagated along higher L-values and after existing from the duct, they penetrated the ionosphere and are trapped in the Earth-ionosphere waveguide. The wave normal at the entrance into the waveguide is such that they propagated towards the equator and are received at a low latitude station Jammu. The mean chorus element parameters observed at Jammu are as follows: lower band frequency $f_{\min }=1.1 \mathrm{kHz}$, upper band frequency $f_{U B}=1.96 \mathrm{kHz}$, frequency sweep rate $d f / d t=1.14 \mathrm{kHz} / \mathrm{s}$ and repetition period $T=2.5 \mathrm{~s}$. A generation mechanism for various temporal and spectral features of recorded VLF chorus emissions is presented on the basis of the recent nonlinear theory. To examine the nonlinear generation mechanism, we have computed the frequency sweep rate of chorus emissions $\partial f / \partial t=2.43 \mathrm{kHz} / \mathrm{s}$ which is very much comparable to our experimentally observed result of $1.14 \mathrm{kHz} / \mathrm{s}$. Thus our results confirm that the nonlinear theory of generation mechanism of chorus emissions is consistent with the analysis of chorus emissions observed at low latitude stations.

\section{Acknowledgments:}

This work is partly supported by ISRO, Bangalore and partly by DST, New Delhi. We are thankful to the reviewer and Editor for their valuable comments and suggestions.

\section{References}

Burtis, W. J. and R. A. Hellewell, Magnetospheric chorus: occurrence patterns and normalized frequency, Planet. Space Sci., 24, 1007, 1976. 
Burton, R. K. and R. E. Holzer, The origin and propagation of chorus in the outer magnetosphere, J. Geophys. Res., 79, 1014, 1974.

Carpenter, D. L. and R. R. Anderson, An ISEE/whistler model of equatorial density in the magnetosphere, J. Geophys. Res., 97, 1097, 1992.

Cornilleau-Wehrlin, N., R. Gendrin, F. Lefeuvre, M. Parrot, R. Grand, D. Jones, A. Bahnsen, E. Vngstrup, and W. Gibbons, VLF electromagnetic waves observed onboard GEOS-1, Space Sci. Review, 22, 371, 1978.

Hattori, K., M. Hayakawa, D. Lagoutte, M. Parrot and F. Lefeuvre, Further evidence of triggered chorus emissions from wavelets in the hiss band, Planet. Space Sci., 39, 1465, 1991.

Hattori, K. and M. Hayakawa, Consideration of dynamic spectra and direction finding results of hiss-triggered chorus emissions, Proc. NIPR Symp. Upper Atmos. Phys., 7, 40,1994.

Hyakawa, M., Study of generation mechanism of magnetospheric VLF/ELF emissions based on the direction findings, Proc. NIPR Symp. Upper Atmos.Phys., 6, 113, 1993.

Helliwell, R. A., Whistlers and Related Ionospheric Phenomena, Stanford. CA. USA, Stanford University Press, 1965.

Helliwell, R. A., A theory of discrete VLF emissions from the magnetosphere, J. Geophys. Res., 72, 4273, 1967.

Helliwell, R. A. and U. S. Inan, VLF wave growth and discrete emissions triggering in the magnetosphere: A feedback-model, J. Geophys. Res., 87, 4427, 1982.

Hikishima, M., S. Yagitani, Y. Omura and I. Nagano, Full particle simulation of whistler-mode rising chorus emissions in the magnetosphere, J. Geophys. Res., 114, A01203, 2009.

Hikishima, M. and Y. Omura, Particle simulations of whistler-mode rising-tone emissions triggered by waves with different amplitudes, J. Geophys. Res., 117, A04226, 2012.

Inan, U. S., Non-linear gyroresonant interactions of energetic particles and coherent VLF waves in the magnetosphere, Ph.D Thesis, Standford Univ. Stanford, Calif., 1977.

Kasahara, Y., H. Uchiyama and Y. Goto, Whistler mode-chorus observed around the plasmapause during magnetic storms, in Frontiers in Magnetospheric Plasma Physics, Celeberating 10 Year of Geotail Operation, edited by M. Hoshino, Y. Omura and L. J. Lanzerotti, P. 228, Elsevier, San Diego, Calif., 2005.

Katoh, Y. and Y. Omura, Study of generation mechanism of VLF triggered emission by selfconsistent particle code, J. Geophys. Res., 111, A12207, 2006.

Katoh, Y. and Y. Omura, Computer simulation of chorus wave generation in the Earth's inner magnetosphere, Geophys. Res. Lett., 34, L03102, 2007.

Lauben, D. S., U. S. Inan, T. F. Bell and D. A. Gurnett, Source characteristics of ELF/VLF chorus, J. Geophys. Res., 107, A12 1429, 2002.

Molving, K., G. Hilfer, R. H. Miller and J. Myczkowski, Self-consistent theory of triggered whistler emissions, J. Geophys. Res., 93, 5665, 1988.

Nunn, D., A self-consistent theory of triggered VLF emissions, Planet. Space Sci., 22, 349, 1974.

Nunn, D., Y. Omura, H. Matsumoto, I. Nagano and S. Yagitani, The numerical simulation of VLF chorus and discrete emissions observed on Geotail satellite using a Vlasov code, $J$. Geophys. Res., 102, 27083, 1997.

Omura, Y. and M. Matsumoto, Computer simulations of basic process of coherent whistler wave-particle interactions in the magnetosphere, J. Geophys. Res., 87, A6 4435, 1982.

Omura, Y. and D. Summers, Computer simulations of relativistic whistler-mode wave-particle interactions, Phys. Plasma, 11, 3530, 2004. 
Omura, Y., N. Furuya and D. Summers, Relativistic acceleration of resonant electrons by coherent whistler mode waves in a dipole magnetic field, J. Geophys. Res., 112, A06236. 2007.

Omura, Y., Y. Katoh and D. Summer, Theory and simulation of the generation of whistler-mode chorus, J. Geophys. Res., 113, A04223, doi: 10.1029/2007JA012622, 2008.

Omura, Y., M. Hikishima, Y. Katoh, D. Summers and S. Yagitani, Nonlinear mechanisms of lower-band and upper-band VLF chorus emissions in the magnetosphere, J. Geophys Res., 114, A07217, doi: 10.1029/2009JA014206, 2009.

Santolik, O. and D. A. Gurnett, Transverse dimensions of chorus in the source region, Geophys. Res. Lett., 30, 1031, doi:10.1029/2002GL016178, 2003.

Santolik, O., D. A. Gurnett, J. S. Pickett, M. Parrot and N. Cornilleau-Wehrlin, A microscopic and nanoscopic view of storm-time chorus on 31 March 2001, Geophys. Res. Lett., 31, L02801, 2004.

Sazhin, S. S. and M. Hayakawa, Magnetospheric chorus emission, Planet. Space Sci., 40, 681, 1992.

Singh, A. K. and K. Ronnmark, Generation mechanism for VLF chorus emissions observed at a low-latitude ground station, Ann. Geophys., 22, 2067, 2004.

Singh, K.K., R. Singh, R. P. Singh and Shyampati, Hisslers: Quasiperiodic VLF noise forms observed at low latitude ground station Jammu ( $L=1.17)$, Geophys. Res. Lett., 31, L19802-02, 2004.

Singh, K.K., J. Singh, R.P. Patel, A.K. Singh, R. P. Singh, R. Singh and P.A. Ganai, Quasiperiodic VLF emission observed during daytime at a low latitude Indian ground station Jammu, J. Earth Syst. Sci., 118, 31, 2009.

Singh, A. K., S.B. Singh and R.P. Patel, An explanation of the observation of whistler-mode chorus emissions at the Indian Antarctic station, Maitri $(\mathrm{L}=4.5)$, Phys. Scr., 81, 035901 (5pp), 2010.

Skoug, R. M., S. Datta, M.P. McCarty and G.K. Parks, A Cyclotron resonance model of VLF chorus emissions detected during electron microburst precipitation, J. Geophys.Res., 101, 21491, 1996.

Smirnova, N. A., Fine structure of the ground observed VLF chorus as an indicator of the wave particle interaction process in the magnetosphere, Planet. Space Sci., 32, 425, 1984.

Stix, T. H., Waves in Plasmas, Am. Inst. of Phys., New York, 1992.

Tao, X., W. Li, J. Bortnik, R.M. Thorne, and V. Angelopoulos, Comparison between theory and observation of the frequency sweep rates of equatorial rising tone chorus, Geophys. Res. Lett., 39, L08106, 2012.

Trakhtengerts, V. Y., Magnetospheric cyclotron maser: backward wave oscillator generation regime, J. Geophys. Res., 100, 17205, 1995.

Trakhtengerts, V. Y., A generation mechanism for chorus emissions, Ann. Geophys., 17, 95, 1999.

Tsurutani, B. T. and E. J. Smith, Post- midnight chorus: a substorm phenomenon, J. Geophys. Res., 79, 118, 1974.

(Received June 21, 2012; revised October 20, 2012; accepted October 29, 2012) 